\title{
Ubenimex, an APN inhibitor, could serve as an anti-tumor drug in RT112 and 5637 cells by operating in an Akt-associated manner
}

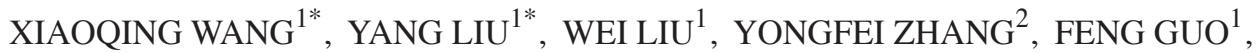 \\ LIJUAN ZHANG $^{1}$, MINGYU CUI ${ }^{1}$, SHUAI LIU ${ }^{3}$ and RONGDE WU ${ }^{1}$ \\ ${ }^{1}$ Department of Pediatric Surgery, Shandong Provincial Hospital \\ Affiliated to Shandong University, Jinan, Shandong 250021; ${ }^{2}$ Department of Dermatology, \\ Shandong Provincial Qianfoshan Hospital Affiliated to Shandong University, Jinan, Shandong 250014; ${ }^{3}$ Department of \\ Urology, Shandong Provincial Hospital Affiliated to Shandong University, Jinan, Shandong 250021, P.R. China
}

Received May 16, 2017; Accepted December 29, 2017

DOI: $10.3892 / \mathrm{mmr} .2018 .8402$

\begin{abstract}
Bladder cancer, a common urinary tract tumor, has high mortality and recurrence rates associated with metastasis. Aminopeptidase N (APN) expression and metastasis have been indicated to be associated with one another. Ubenimex may function as an APN inhibitor to inhibit the degradation of the extracellular matrix during tumorigenesis. Furthermore, APN has been widely used as an adjuvant therapy for the treatment of tumors; however, little information is available regarding the impact of ubenimex on patients. Autophagy is suggested to be important in the transformation and progression of cancer. Additionally, apoptosis, which leads to the rapid demolition of cellular organelles and structures, has also been suggested as an important factor. Thus, the present study investigated the role of ubenimex in inhibiting migration and invasion by downregulating APN expression levels to induce autophagic cell death and apoptosis in bladder cancer cells. RT112 and 5637 cell lines were treated with varying doses of ubenimex. Cell viability was measured by CCK 8 colorimetry and flow cytometry. Using fluorescence microscopy, autophagic cell death was assessed using acridine orange/ethidium bromide staining. Furthermore, apoptotic cell death was assessed using flow cytometry and Trypan blue staining was used to evaluate
\end{abstract}

Correspondence to: Professor Rongde Wu, Department of Pediatric Surgery, Shandong Provincial Hospital Affiliated to Shandong University, 324 Jingwu Street, Jinan, Shandong 250021, P.R. China

E-mail:wrd2190@163.com

Dr Shuai Liu, Department of Urology, Shandong Provincial Hospital Affiliated to Shandong University, 324 Jingwu Street, Jinan, Shandong 250021, P.R. China

E-mail: 15866600623@163.com

${ }^{*}$ Contributed equally

Key words: ubenimex, autophagic cell death, aminopeptidase N, apoptosis, Akt signaling pathway the cell death rate. Protein expression was determined by western blot analysis. Matrigel invasion assays were exploited to assess the invasion capabilities of 5637 cells. Wound-healing migration assays and Matrigel migration assays were exploited to assess the migratory abilities of 5637 cells. Treatment with ubenimex was accompanied by decreased Akt expression, indicating that ubenimex may have similar functions to Akt inhibitors. Results also indicated that ubenimex inhibited cell migration and invasion in bladder cancer cells. Furthermore, ubenimex also induced autophagic cell death and apoptosis, which suggested that mixed programmed cell death occurred in ubenimex-treated bladder cancer cells. The results from the present study suggest that ubenimex may be a potential adjuvant therapy for the treatment of bladder cancer.

\section{Introduction}

Bladder cancer is the most common urological tumor and ranks as the leading cause of death in patients with urinary tract disease (1). Despite the development of treatment strategies, within the first year after TURP (transurethral resection of prostate) treatment of NMIBC (non-muscle invasive bladder cancer), the probability of disease recurrence ranges from 15 to $60 \%$ and the probability of disease progression at 5 years ranges from 7 to $40 \%$ (2). Furthermore, MIBC has a higher rate of progressing to distant, life-threatening metastases than NMIBC and is still the major cause of low survival rate. The high metastatic rate of MIBC has always been the main obstacle in clinical treatment (3). Improved treatment requires a detailed understanding of urothelial carcinoma pathogenesis and molecular biology. Therefore, what we urgently need is the suppression of bladder cancer invasion and metastasis and the development of an adjuvant therapy for bladder cancer.

Aminopeptidase N (APN) is also known as the cell surface molecule CD13. It is involved in various cellular processes, including cell cycle control, cell differentiation, cell motility, angiogenesis, cellular attachment and invasion/metastasis of various malignancies, including bladder cancer (4). APN/CD13 is overexpressed in several cancers, including pancreatic cancer, non-small cell lung cancer and colon cancer. In the cancers mentioned above, APN levels positively correlate with 
tumor metastasis (5). Although the correlation between APN expression and invasion/metastasis of cancer is increasingly clear, little is known about the role of APN/CD13 in the invasion and metastatic ability of bladder cancer cells.

Ubenimex has been exploited as an adjuvant therapy to enhance the effects of antitumor treatments after surgery. It has been widely used in the treatment of leukemia, non-small cell lung cancer, gastric cancer and cervical cancer (6-8). Our previous study demonstrated that ubenimex functions as an antitumor drug in prostate cancer cells. We also demonstrated that the inhibition of APN activity and the resulting induction of cell death plays a key role during this process (9). Apoptosis, or caspase-dependent cell death, is a type of programmed cell death (PCD) that can dispose of damaged cells (10). Autophagy, or caspase-independent cell death, also plays a crucial role in tumor cell death (11). Akt is a classical signaling pathway that participates in the regulation of tumor cell apoptotic and autophagic cell death (12). Thus, we propose that ubenimex triggers mixed PCD in bladder cancer. In this study, we determine the effects of ubenimex on bladder cancer cells and the potential underlying mechanisms.

\section{Materials and methods}

cell culture. The RT112 and 5637 cell lines were purchased from the Cell Bank of the Chinese Academy of Sciences. Cells were maintained in RPMI-1640 medium (BioInd, Kibbutz Beit Haemek, Israel) supplemented with $1 \%$ penicillin, streptomycin and $10 \%$ fetal bovine serum (FBS; BioInd). The cells were incubated at $37^{\circ} \mathrm{C}$ in a humidified atmosphere with $5 \% \mathrm{CO}_{2}$.

CCK-8 cytotoxicity assay. RT112 and 5637 cells in an exponential phase of growth were harvested and seeded into 96-well plates at a density of 15,000 cells/well (RT112) and 10,000 cells/well (5637) in RPMI-1640 medium supplemented with different concentrations of ubenimex or/and 3MA (control, $0.1 \mathrm{mg} / \mathrm{ml}, 0.25 \mathrm{mg} / \mathrm{ml}, 0.5 \mathrm{mg} / \mathrm{ml}, 1 \mathrm{mg} / \mathrm{ml}$, $1 \mathrm{mg} / \mathrm{ml}+3 \mathrm{MA})$. Another treatment groups were divided into four parts (control, $0.5 \mathrm{mg} / \mathrm{ml} \mathrm{UB}$, Akt stimulator, $0.5 \mathrm{mg} / \mathrm{ml}$ $\mathrm{UB}+\mathrm{Akt}$ stimulator (SC-79, melonepharma, Dalian, China). After 16, 24, 36 and $48 \mathrm{~h}$ of culture, $10 \mu \mathrm{l}$ CCK-8 solution (CCK-8 cytotoxicity assay kit; EnoGene Biotech, Co., Ltd., Nanjing, China) was added into each well. The plates were then incubated for an additional $4 \mathrm{~h}$ at $37^{\circ} \mathrm{C}$, and the absorbance was determined using a microplate reader (EL340; Bio-Tek Instruments, Hopkinton, MA, USA) at $450 \mathrm{~nm}$.

Wound healing migration assays. 5637 cells were plated in 6 -well culture plates and grown to $90 \%$ confluency. Next, a sterile P200 pipette tip was used to create a scratch across the monolayer. Cell debris was removed by washing with phosphate-buffered saline (PBS), and the cells were cultured in RPMI-1640 medium without FBS supplemented with different concentrations of ubenimex. The area of the scratch was measured at 16 and $24 \mathrm{~h}$. Quantification was performed by measuring the area of cell migration at different time points compared to the scratched area at $0 \mathrm{~h}$.

Matrigel invasion assay and matrigel migration assay. Invasion assays were performed using transwell chambers and
5637 cells were used. Control untreated cells or cells treated with ubenimex $(0.5$ or $1 \mathrm{mg} / \mathrm{ml}$ for $16 \mathrm{~h})$ were trypsinized, and $1 \times 10^{5}$ cells were plated in the upper wells in serum-free medium, while medium with $10 \% \mathrm{FBS}$ was added to the lower well as a stimulus. After $20 \mathrm{~h}$ of incubation, the cells on the Matrigel side of the chambers were removed using a cotton swab. The inserts were fixed in methanol and stained using hematoxylin and eosin (H\&E) staining. The number of invading cells attached to the other side of the inserts was quantified under a light microscope using 6 random fields at a magnification of $x 200$. The experiment was performed in triplicate. 5637 was chosen to form the invasion assay. In addition, invasion assays were performed using transwell chambers that were pre-coated with $40 \mu \mathrm{l}$ of $1 \mathrm{mg} / \mathrm{ml}$ Matrigel matrix (BD Biosciences, Franklin Lakes, NJ, USA, USA). We controlled each group contains $2.5 \times 10^{5}$ cells for plated in the upper wells, the remaining steps followed as Matrigel migration assay.

Acridine orange (AO)/ethidium bromide $(E B)$ double staining. Cells were cultured in 6-well plates for $24 \mathrm{~h}$, and were treated with different doses of ubenimex $(0,0.5$ and $1 \mathrm{mg} / \mathrm{ml})$ for $16 \mathrm{~h}$. After the indicated treatment times, $\mathrm{AO} / \mathrm{EB}$ operating fluid was mixed reagent $A$, reagent $B$, and reagent $C$ at a certain rate of 1:1:8. Each sample was added 2 ul AO/EB operating fluid, discarded the supernatant after low speed centrifugal $(500 \mathrm{~g} / \mathrm{min})$, resuspend cells in $\mathrm{AO} / \mathrm{EB}$ dilution buffer at a concentration of $5 \times 10^{6}$ cells $/ \mathrm{ml}$, then add $1 \mathrm{ul} \mathrm{AO} / \mathrm{EB}$ operating fluid to $25 \mathrm{ul}$ cell suspension. Next, the cells were observed under a fluorescence microscope (Nikon Corporation, Tokyo, Japan).

Western blot analysis. To determine APN, LC-3, Beclin 1, Akt/P-Akt, P62, C-caspase-3/P-caspase-3 levels, proteins were extracted from the cells by suspension in radioimmunoprecipitation assay (RIPA) buffer. Samples were centrifuged at $12,000 \mathrm{rpm}$ at $4^{\circ} \mathrm{C}$ for $30 \mathrm{~min}$, and the supernatants were recovered for analysis. The protein concentrations were determined using the Bradford protein method and the bicinchoninic acid (BCA) protein assay kit (Sigma-Aldrich; Merck KGaA, Darmstadt, Germany). Protein (40 $\mu \mathrm{g})$ was electrophoresed on a pre-cast bis-Tris polyacrylamide gel (12\%), and then transferred onto a polyvinylidene difluoride (PVDF) membrane. Membranes were blotted with rabbit anti-APN $(1: 1,000)$, rabbit anti-LC3B (1:500), rabbit anti-beclin 1 (1:1,000), rabbit anti-Akt $(1: 1,000)$, rabbit anti-p-Akt $(1: 1,000)$, rabbit anti-P62 (1:1,000), rabbit anti-Caspase-3 (1:1,000) (all from Abcam, Cambridge, MA, USA) and mouse anti-actin (1:1,000; ProteinTech Group, Inc., Chicago, IL, USA), followed by horseradish peroxidase (HRP)-conjugated secondary antibodies (1:5,000; ZsBio, Beijing, China). Immunoblots were visualized using enhanced chemiluminescence (LAS-4000).

Cell apoptosis analysis by flow cytometer. Apoptosis was measured by Annexin V-FITC/PI dual staining. Cells were cultured in a petri dish for $36 \mathrm{~h}$, and then were treated with different doses of ubenimex $(0,0.5$ and $1 \mathrm{mg} / \mathrm{ml})$ for $16 \mathrm{~h}$. After the indicated treatment times $(16 \mathrm{~h})$, the cells in an exponential phase of growth were harvested. We divided RT112 and 5637 cells into control group, PE group, 7-AAD group and treatment group. Then wash cells twice with cold PBS 
and resuspend cells in $1 \mathrm{X}$ Binding buffer at a concentration of $1 \times 10^{6}$ cells $/ \mathrm{ml}$, transfer $100 \mu \mathrm{l}$ of the solution ( $1 \times 10^{5}$ cells) to a $5 \mathrm{ml}$ culture tube. Add $5 \mu \mathrm{l}$ of PE Annexin V and $5 \mu 1$ 7-AAD. Gently vortex the cells and incubate for $15 \mathrm{~min}$ at $\mathrm{RT}\left(25^{\circ} \mathrm{C}\right)$ in the dark. Add $400 \mu \mathrm{l}$ of $1 \mathrm{X}$ Binding buffer to each tube. Finally, the samples were analyzed by flow cytometry within $1 \mathrm{~h}$ on a FACSCalibur (BD Biosciences, Franklin Lakes, NJ, USA).

Trypan blue staining. The cells were cultured in six-well plates for $24 \mathrm{~h}$, which is divided into four groups (control, $0.5 \mathrm{mg} / \mathrm{ml}$ $\mathrm{UB}, 0.5 \mathrm{mg} / \mathrm{ml} \mathrm{UB}+3 \mathrm{MA}, 0.5 \mathrm{mg} / \mathrm{ml} \mathrm{UB}+\mathrm{Akt}$ stimulator). The groups were treated for $24 \mathrm{~h}$. After the indicated treatment times, cell suspension and $0.4 \%$ trypan blue solution mixed with 9:1. In three min, with the counting plate, live cells and dead cells were counted. Cell vitality were measured under the following formula: Living cell rate $(\%)=$ living cell total/(total number of living cells + dead cells) x $100 \%$.

Statistical analysis. The data were statistically analyzed with Student's t-test, $\chi^{2}$ or Fisher's exact tests using SPSS version 19.0 (IBM SPSS, Armonk, NY, USA). P<0.05 was considered to indicate a statistically significant difference.

\section{Results}

Ubenimex inhibits APN expression in bladder cancer cells. We investigated the expression of APN/CD13 and the involvement of the Akt signaling pathway in bladder cancer cells following treatment with ubenimex by western blot analysis (Fig. 1). Both RT112 and 5637 cells were treated with ubenimex for $16 \mathrm{~h}$. Inhibition of APN was significant and correlated with ubenimex dosage. Thus, we concluded that ubenimex functions as an APN inhibitor in bladder cancer cells. Therefore, the findings underline that ubenimex functions as an APN inhibitor in bladder cancer cells.

Ubenimex induces autophagic cell death in bladder cancer cells. AO-EB staining and western blot analyses were used to examine the level of autophagy in bladder cancer cells. According to the results of a CCK8 cytotoxicity assay, we used cells treated for $16 \mathrm{~h}$ with ubenimex for study. In addition, we observed that ubenimex increased the expression of LC3B and Beclin 1 and decreased the expression of P62 in both RT112 and 5637 cells (Fig. 1). This finding indicates that a higher level of autophagic cell death was induced by higher doses of drug. Moreover, AO-EB staining can be a reflection of the extent of DNA damage, which indicates autophagic cell death (13). AO staining levels vary depending on ubenimex dosage in the bladder cancer cells: Staining levels increased with the dose of ubenimex (Fig. 2). In addition, combined treatment of ubenimex and 3-methyladenine (an inhibitor of autophagy) increased viability compared with ubenimex treatment alone (Fig. 3A and B). Therefore, the treatment of ubenimex was shown to induce autophagic cell death in bladder cancer cells.

Ubenimex inhibits the proliferation of bladder cancer cell lines. To assess the effects of ubenimex on the proliferation of bladder cancer cells, we used the CCK8 cell proliferation assay and measured viability after 16, 32, and $48 \mathrm{~h}$ (Fig. 3A and B).
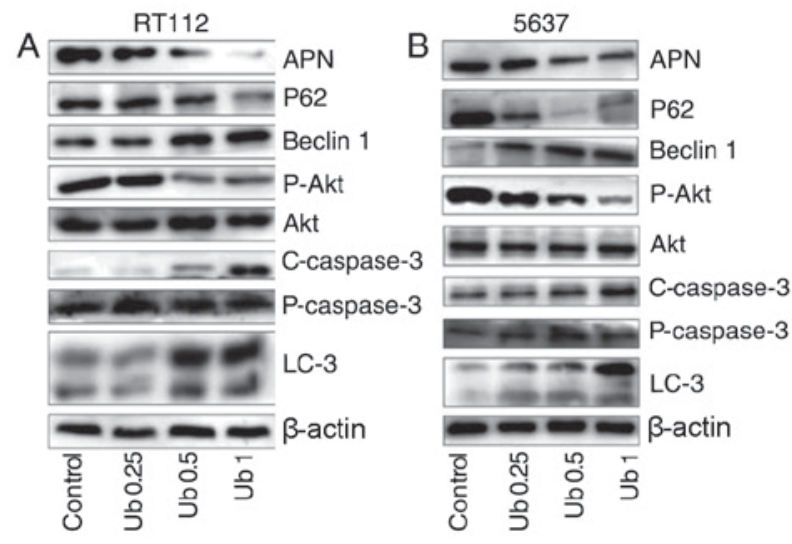

Figure 1. Ubenimex promotes cell death and inhibits the expression of APN and Akt in bladder cancer cells. (A and B) Western blotting showing the expression of APN, LC-3, Beclin 1, P62, P-Akt, Akt, C-caspase-3, and P-caspase-3 in RT112 and 5637 cells after treatment for $16 \mathrm{~h}$ with ubenimex $(0.25,0.5$ or $1 \mathrm{mg} / \mathrm{ml})$. $\beta$-actin is shown as a loading control. Levels of LC-3, Beclin 1 and P62 changed in a dose-dependent manner, with a trend mirroring that of autophagy levels. Western blotting of P-Akt and Akt expression in RT112 and 5637 cells after treatment for $16 \mathrm{~h}$ with ubenimex $(0.25,0.5$ or $1 \mathrm{mg} / \mathrm{ml})$. Data are expressed as the mean \pm standard deviation of 3 independent experiments. C-caspase-3, cleaved-caspase-3; P-caspase-3, pro-caspase-3.

RT112 and 5637 cells were treated with ubenimex as mentioned previously. Both bladder cancer cell lines demonstrated a prominent, dose-dependent decrease in cell viability with ubenimex treatment, though the effect was more obvious in the RT112 cell line. In summary, proliferation of bladder cancer cell lines was markedly decreased after treatment with ubenimex.

Ubenimex induces apoptosis in bladder cancer cells. Western blot analyses and flow cytometry were used to examine apoptosis levels in the bladder cancer cell lines. Ubenimex induced apoptosis in a significantly higher percentage of treated cells compared to the control groups. For further experiments, we treated bladder cancer cells with ubenimex for a $16 \mathrm{~h}$ period. Next, we demonstrated that ubenimex enhanced the expression of caspase- 3 in bladder cancer cells. Flow cytometry was used to assess the proportion of cells undergoing early apoptosis and autophagy in RT112 and 5637 cells with the treatment of ubenimex (Fig. 4). The quantitative results showed that ubenimex significantly induced early apoptosis and autophagic cell death in a dose-dependent manner in RT112 and 5637 cells. It also had shown a steady increasing trend with the increased dose of ubenimex.

Ubenimex inhibits migration and invasion in bladder cancer cell lines. As indicated in Figs. 5 and 6, the migration and invasion capacity of 5637 cells using wound-healing migration and transwell assays were assessed, respectively. We expected that the decreased migratory capacity of the 5637 cells was due to ubenimex treatment after 16 or more h of exposure (Figs. 5A and 6A). Obviously, the increased dose of ubenimex also plays a key role in inhibiting the migration ability of bladder cancer cells. Furthermore, we investigated the effect of ubenimex on the invasive activity of 5637 cells using a transwell cell-culture chamber coated with Matrigel. As shown in (Fig. 5C), the invasion ability of 5637 cells was strongly suppressed by 

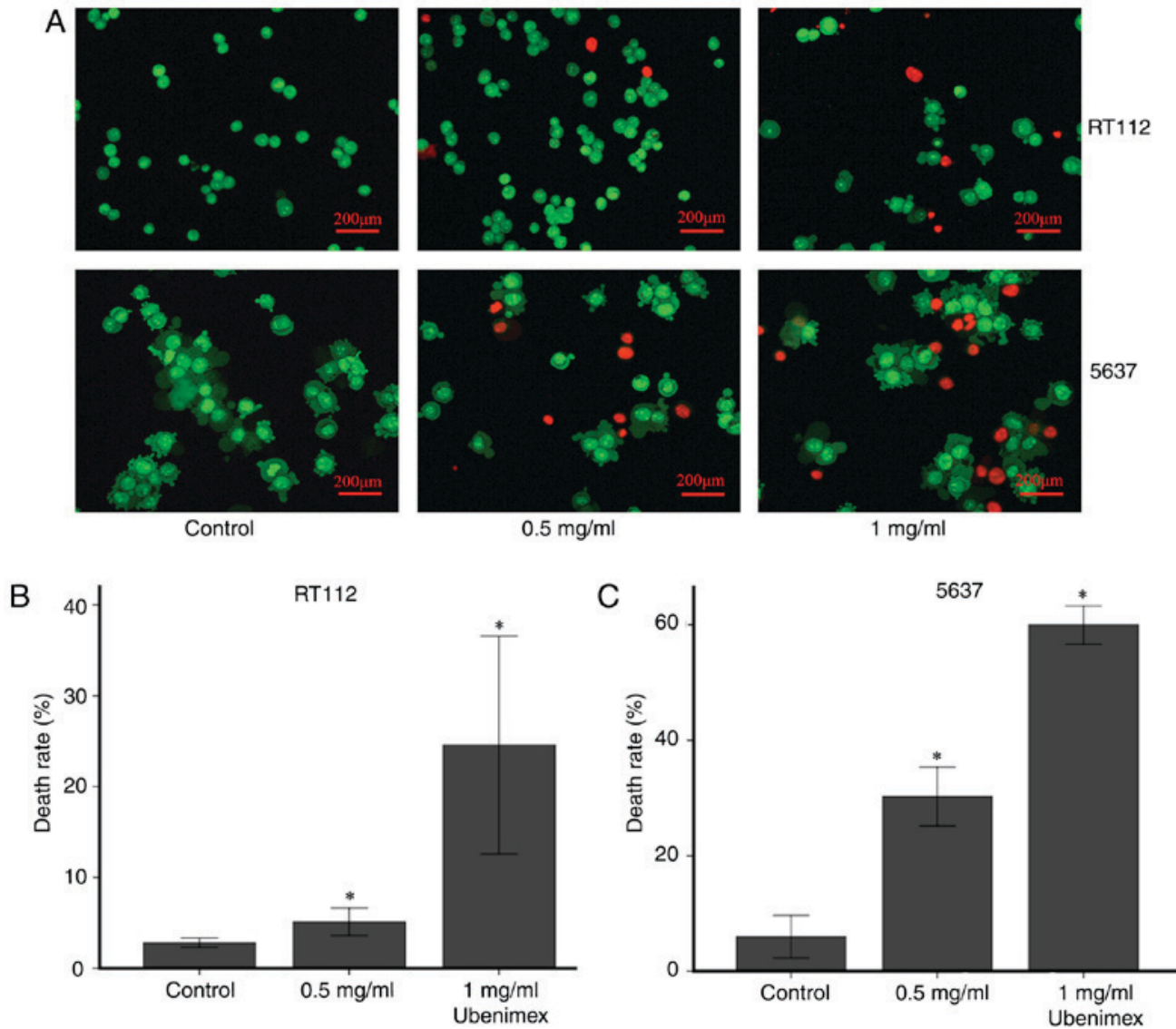

Figure 2. Ubenimex induces cell death in both RT112 and 5637 bladder cancer cells. (A) RT112 and 5637 cells were treated with different doses of ubenimex $(0.5$ and $1 \mathrm{mg} / \mathrm{ml})$. After $16 \mathrm{~h}$, fluorescence microscopy was used to examine DNA damage. The death rates of (B) RT112 and (C) 5637 cells per field of a high-power microscope as determined by acridine orange staining are shown. ${ }^{*} \mathrm{P}<0.05$ vs. the control. Data are expressed as the mean \pm standard deviation of 3 independent experiments.
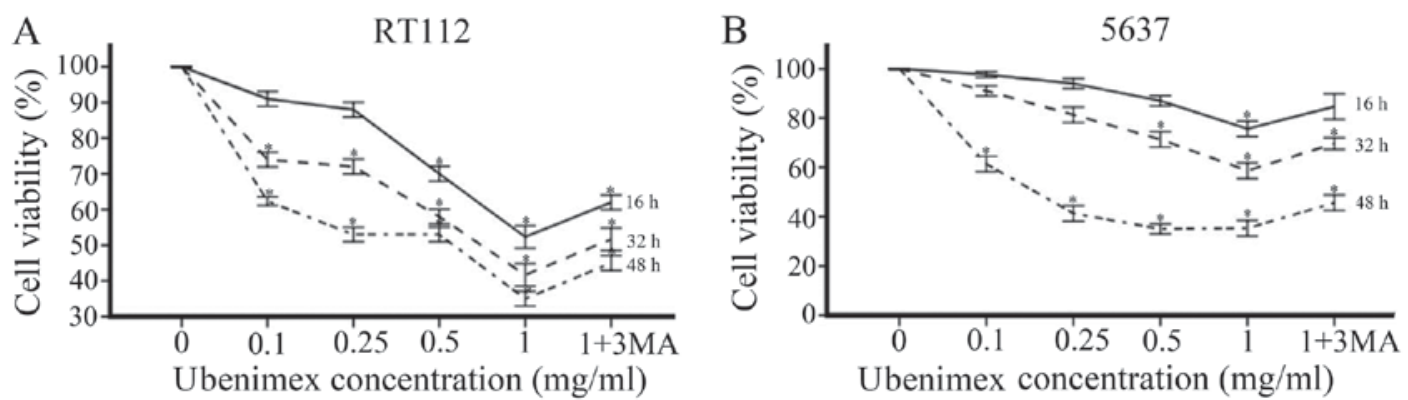

Figure 3. Cell viability was inhibited and cell death was induced by ubenimex in both RT112 and 5637 cells. (A and B) CCK- 8 cytotoxicity assays were performed after culturing RT112 or 5637 cells for 16,32 or $48 \mathrm{~h}$ with the indicated concentrations of ubenimex. "P $<0.05$ for ubenimex treated cells compared with untreated control cells. Ubenimex+3MA was also tested. "P $<0.05$ for cells treated with $1 \mathrm{mg} / \mathrm{ml} \mathrm{UB}+3 \mathrm{MA}$ vs. $1 \mathrm{mg} / \mathrm{ml}$ UB treatment alone. Data are expressed as the mean \pm standard deviation of 3 independent experiments. UB, ubenimex; 3MA, 3-Methyladenine.

ubenimex. Thus, we demonstrated that ubenimex treatment suppressed the migration and invasion of bladder cancer cells.

Ubenimex treatment effects involve the Akt signaling pathway. Previous studies have shown that the Akt signaling pathway is involved in the regulation of autophagy and apoptosis (12). Stress is capable of activating the Akt signal transduction pathway in tumor cells, stimulating cell-protective mechanisms $(14,15)$. As mentioned above, to evaluate the involvement of the Akt signaling pathway in the effects of ubenimex treatment, we performed a western blotting to detect the expression of protein phosphorylation. High doses of ubenimex treatment decreased the phosphorylation of Akt compared with the control group. In order to assess the involvement of Akt, we introduced Akt stimulator into treated group to assess the changing trend of Akt (Fig. 7). Both trypan blue staining and CCK-8 cytotoxicity assay were used to estimate the rate of cell death or cell viability after the introduction of Akt-stimulator. Upon Akt activation, cell death decreased and a distinct cell viability change was noticed. (Fig. 7A, B and D). We speculated that ubenimex plays a role as an Akt agonist. These findings suggest that ubenimex strongly induces both apoptotic and autophagic cell death. 

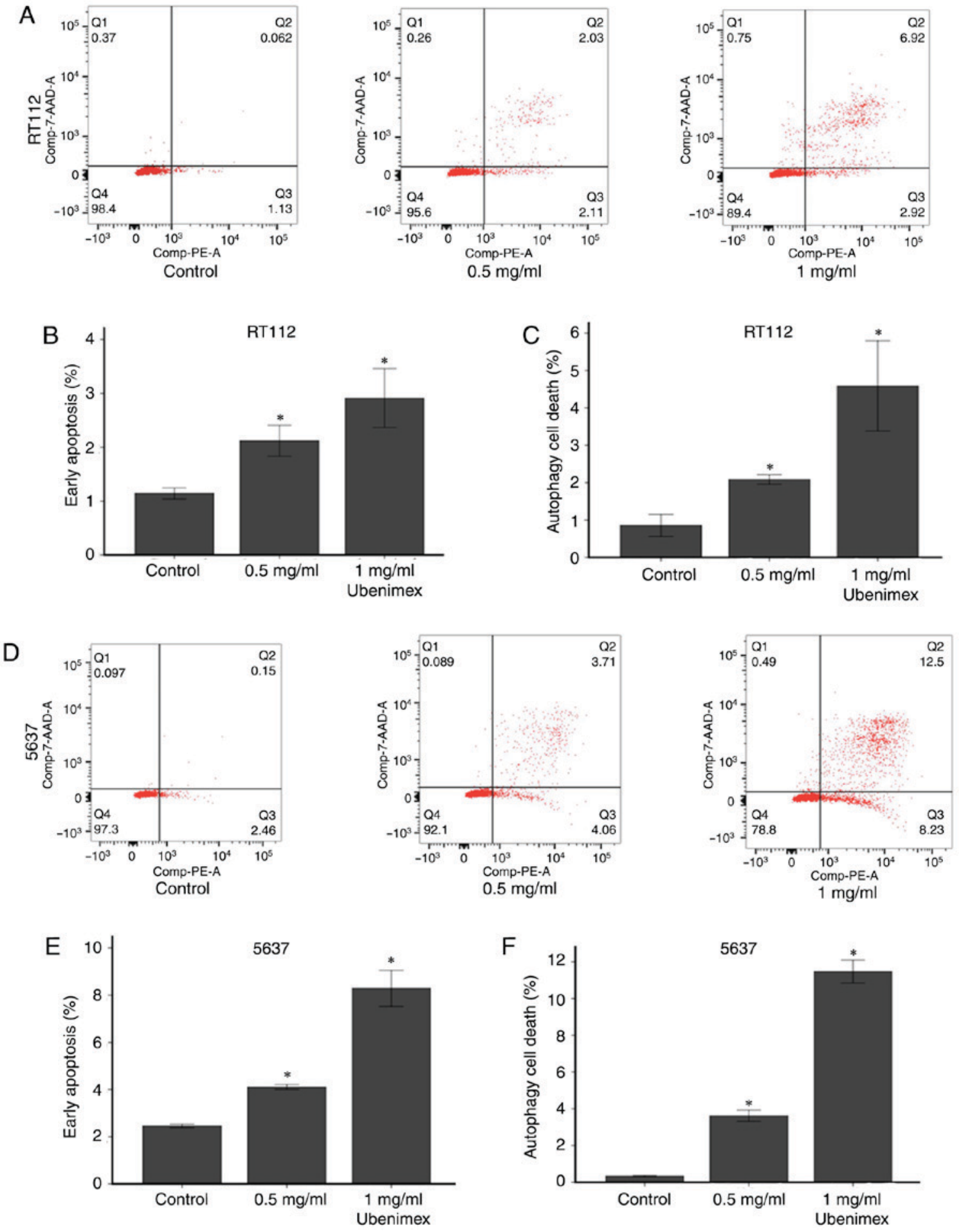

Figure 4. RT112 and 5637 cells were treated with ubenimex $(0.5$ and $1 \mathrm{mg} / \mathrm{ml})$ for $16 \mathrm{~h}$ and then fluorescein isothiocyanate-stained cells were detected using flow cytometry. (B and E) Early apoptosis (both RT112 and 5637) was detected using an annexin V apoptosis detection kit and measured using flow cytometry. (A and D) Cell debris is PE Annexin V negative and 7-AAD positive (Q1); cells that are dead or in late apoptosis are both PE Annexin V and 7-AAD positive (Q2); cells that are in early apoptosis are PE Annexin V positive and 7-AAD negative (Q3); cells that are considered viable are PE Annexin V and 7-AAD negative (Q4). " $\mathrm{P}<0.05$ vs. the control. (C and F) Autophagic cell death (both RT112 and 5637) was also measured using flow cytometry. ${ }^{*} \mathrm{P}<0.05$ vs. control. Data are presented as the mean \pm standard deviation of three independent experiments.

\section{Discussion}

In our previous and current studies, we found ubenimex is sufficient to induce prostate cancer cells and bladder cancer cells death, and at the same time the level of autophagy increased. With the help of literature review, we found that ubenimex have suppressive effect on proliferation of leukemic cell line (16). The process contained the induction of apoptosis by ubenimex (17). Ubenimex also could inhibit cell proliferation, migration and invasion in renal cell carcinoma, which of the effect is autophagy-associated (18). Furthermore, ubenimex synergistically enhances the effects 


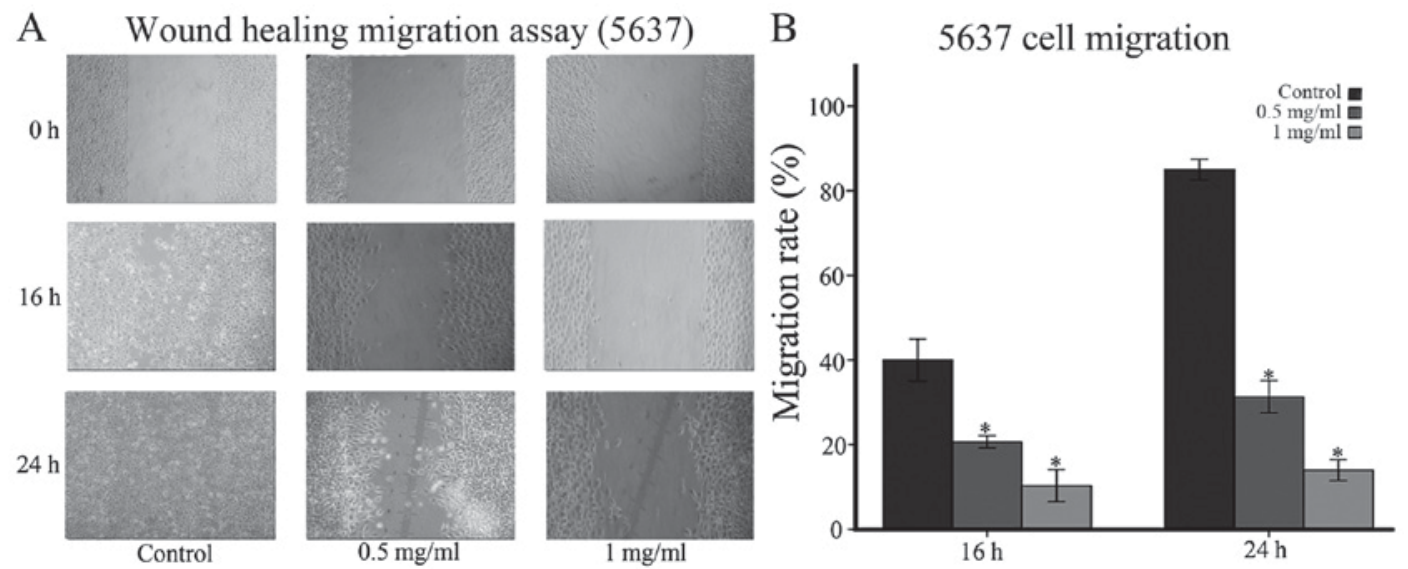

Figure 5. Ubenimex reduces the cell migration and invasion capacity of 5637 cells. Transwell migration and invasion assays were performed to determine the migration and invasion of the 5637 cells. (A) The images above are shown after culture for the indicated times in control medium or different doses of ubenimex, as described above. (B) Quantification was performed by counting the number of cells that passed through the small well using a high-power microscope. These values are expressed as the mean \pm standard deviation of 3 independent experiments. ${ }^{*} \mathrm{P}<0.05$ vs. the untreated control cells. Data are expressed as the mean \pm standard deviation of 3 independent experiments.

A
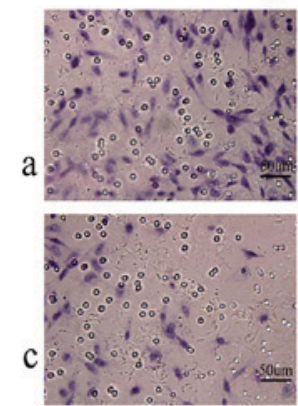

$\mathrm{B}$

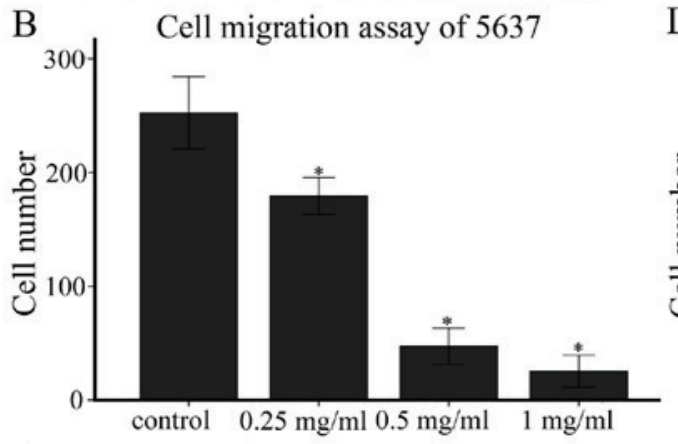

$\mathrm{C}$

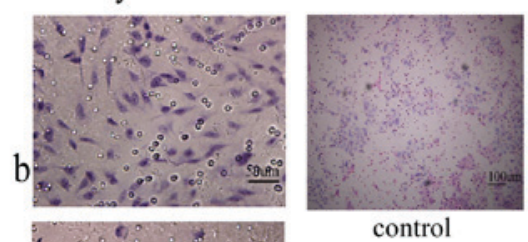

control
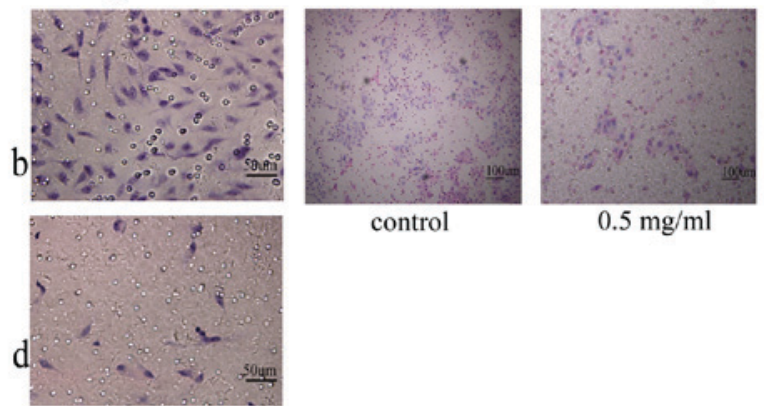

$0.5 \mathrm{mg} / \mathrm{ml}$

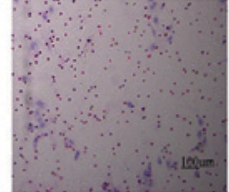

$1 \mathrm{mg} / \mathrm{ml}$
D Cell invasion assay of 5637

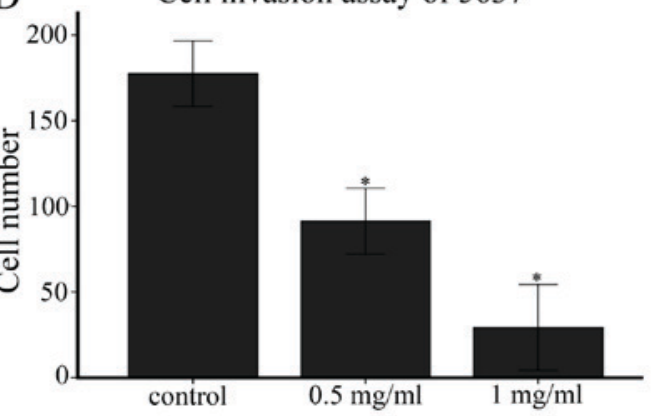

Figure 6. Ubenimex reduced 5637 cell migration capacity. (A and B) Wound healing migration assays were performed to determine the motility of the 5637 cells. (C) The images above are shown after the indicated length of culture in control medium or medium with $0.5 \mathrm{or} 1 \mathrm{mg} / \mathrm{ml}$ ubenimex. (D) Quantification was performed by measuring the scratch area at different time points after migration compared to the scratched area at $0 \mathrm{~h}$. "P<0.05 vs. the untreated control cells. Data are expressed as the mean \pm standard deviation of 3 independent experiments.

of anticancer drugs in hepatocellular carcinoma, osteosarcoma cell lines and human tumor-derived cell lines (19-21). Then we assumed that ubenimex may have a general effect on different cancer types.

Based on the decrease in APN expression, ubenimex could be a potential treatment for the suppression of metastasis and invasion in bladder cancer cells. APN expression has been confirmed to be involved in various cellular processes, including cellular attachment, motility and invasion/metastasis of various malignancies (22). The inhibition of APN expression results in a significant decrease in the metastasis of T24 cell lines (4). High APN expression has been considered an indicator of metastasis and poor prognosis in bladder cancer, as well as in lung, pancreas and colon cancers $(5,23,24)$. Moreover, a previous study revealed that ubenimex could inhibit metastasis and invasion of PC-3 cells and that this effect was related to the inhibition of APN activity (9). Thus, we analyzed ubenimex-treated RT112 and 5637 cells by western blotting and found that the expression of APN was significantly decreased compared to the control group. Furthermore, we showed by Matrigel invasion, Matrigel migration and wound-healing migration assays that the migratory and 
A
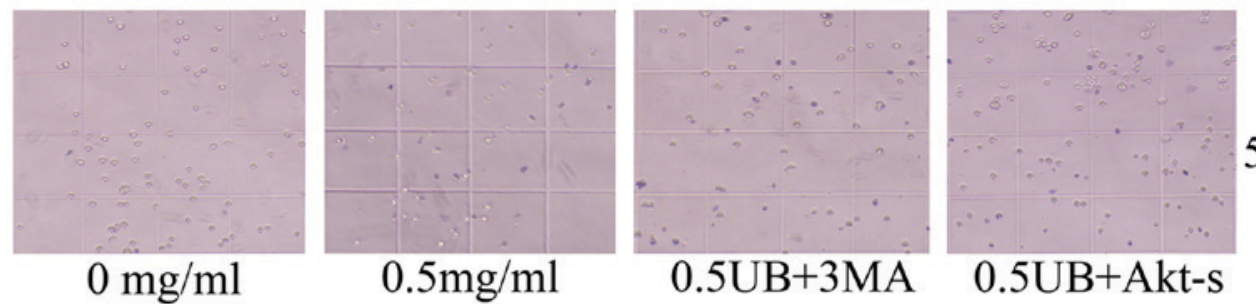

5637

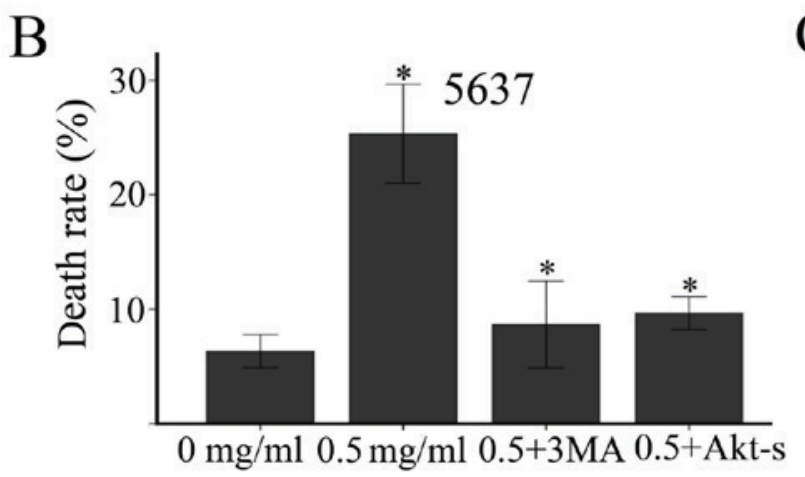

$\mathrm{C}$
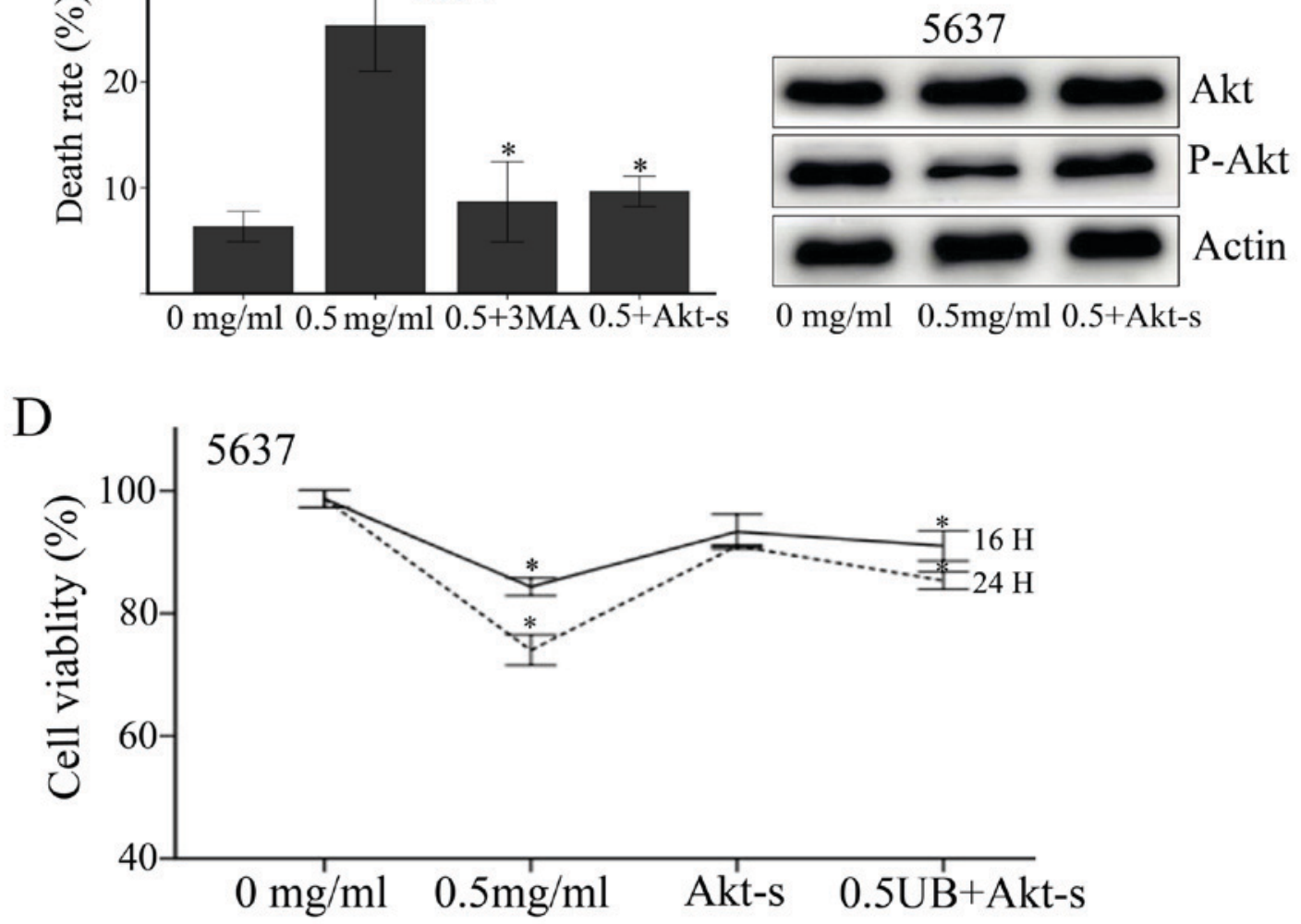

Figure 7. Trypan blue staining was performed to determine the role of Akt stimulator. (A) The cells are divided into four groups (control, $0.5 \mathrm{mg} / \mathrm{ml} \mathrm{UB}$, $0.5 \mathrm{UB}+3 \mathrm{MA}, 0.5 \mathrm{UB}+10 \mathrm{ug} / \mathrm{ml}$ Akt stimulator). (B) Ubenimex promoted cell death as expected. Furthermore, 3MA and Akt stimulator reversed the toxic effects of ubenimex, significantly increasing the cell survival rate above that of the ubenimex treatment group. ${ }^{*} \mathrm{P}<0.05$, for cells treated with $0.5 \mathrm{UB}+\mathrm{Akt}$ stimulator and $0.5 \mathrm{UB}+3 \mathrm{MA}$ vs. $0.5 \mathrm{mg} / \mathrm{ml}$ ubenimex treatment alone. CCK-8 cytotoxicity assay was performed to determine the role of Akt stimulator. The cells are divided into four groups (control, $0.5 \mathrm{mg} / \mathrm{ml} \mathrm{UB}$, Akt stimulator, $0.5 \mathrm{UB}+\mathrm{Akt}$ stimulator). After $16 \mathrm{~h}$ and $24 \mathrm{~h}$ culture of 5637 cells with the indicated concentrations of ubenimex and Akt stimulator $(10 \mathrm{ug} / \mathrm{ml}$ ), (D) CCK-8 cytotoxicity assays revealed that cell viability increased with Akt stimulator treatment compared with the $0.5 \mathrm{mg} / \mathrm{ml}$ ubenimex treatment group. (C) Western blotting confirmed this result. "P<0.05, for cells treated with $0.5 \mathrm{mg} / \mathrm{ml} \mathrm{UB}+\mathrm{Akt}$ stimulator vs. $0.5 \mathrm{mg} / \mathrm{ml}$ ubenimex treatment alone. Data are expressed as the mean \pm standard deviation of 3 independent experiments. UB, ubenimex; $3 \mathrm{MA}$, 3-Methyladenine; Akt-s, Akt stimulator.

invasion capacity of 5637 cells was decreased with ubenimex treatment. Thus, we conclude that ubenimex functions as an APN inhibitor to inhibit metastasis and invasion in bladder cancer cells. In several cancer cell lines, the mechanism of overexpress or knock-down APN has been explored in multimolecular process regulating cell migration $(25,26)$. To a certain extent, these prove that there exist relations between reduced expression of APN and decreased migration/invasion. However, in bladder cancer cells, this paper didn't go deep enough on it, and this part will be gradually improved in our future research.

Autophagy is a dynamic recycling system which relies on lysosomal proteolysis to maintain cellular homeostasis. However, excessive autophagy can lead to cell death (27). Autophagy directly takes part in many types of physiological processes, including metabolism, stress responses, and cell death pathways in cancer cells (28). However, the roles of autophagy in bladder cancer need to be further clarified. In our previous study, we showed that autophagic cell death in prostate cells could be induced by ubenimex (9). In the current study, we demonstrate similar effects in bladder cancer cells. This is the first attempt to study effects of ubenimex treatment on the regulation of autophagy in bladder cancer cells. In our study, we used western blotting to examine the level of autophagy in this context, while AO/EB staining was used to show the amount of cell death related to the doses of ubenimex. Positive results indicated that ubenimex induces autophagic cell death in bladder cancer cells and that this induction occurred in a dose-dependent manner. Furthermore, as both the CCK-8 cell cytotoxicity assay and AO/EB staining 
demonstrated a correlation between cell death and dosage, we considered that autophagic cell death may participate in the cell death program. As it turned out, 3MA (3-Methyladenine, an inhibitor of autophagy) partially reversed the beneficial effects induced by ubenimex: Cell death was significantly reduced when compared to the ubenimex only treatment group. Thus, we maintain that ubenimex induces autophagic cell death in bladder cancer cells.

Apoptosis plays an extensively investigated role whereby cells undergo a caspase-dependent 'self-killing' mechanism. The activation of catabolic enzymes leads to rapid demolition of cellular structures and organelles, eliminating damaged or aged cells and organelles (10). In our study, the apoptosis of bladder cancer cells was assessed by Annexin V-PE/7AAD staining and western blotting. Analysis of flow cytometric results showed that apoptosis of RT112 and 5637 cells increased with ubenimex treatment. Western blotting also demonstrated that caspase-3 was increased in this context. Thus, we detected a mixed phenotype of apoptosis and autophagic cell death in response to ubenimex treatment in bladder cancer cells. However, further study is necessary to elucidate the mechanisms of apoptosis and autophagy activation in bladder cancer. A literature review showed many examples of a complicated relationship between apoptosis and autophagy (10).

The Akt signaling pathway plays a crucial role in the regulation of both apoptosis and autophagy, involving many growth factors and receptors $(29,30)$. The activation of the Akt pathway acts as a survival signal, preventing cells from undergoing apoptosis (31). In oral cancer and colorectal cancer, the induction of autophagy and apoptosis is associated with the disruption of Akt signaling $(32,33)$. Previous literature indicates that some signal transduction pathways triggered by common cellular stresses can trigger both autophagic cell death and apoptosis (10). In our present study, the addition of ubenimex significantly induced apoptotic and autophagic cell death accompanied by a decrease in Akt expression. It was demonstrated that ubenimex might activate mixed PCD concurrent with the inhibition of the Akt signaling pathway.

We expect that additional clinical insights may be obtained from bladder cancer cells. They are valuable in the study of early postoperative bladder irrigation after treatment of a partial cystectomy as well as transurethral resection in early-stage bladder cancer. Experiments in vitro may be a fairly good approximation of postoperative bladder irrigation because bladder cancer cells and drugs make reciprocal contacts in this environment. Combined infusions of ubenimex and BCG or mitomycin may be more practical in the clinic. Ubenimex has already been used in medical treatments, but its role as an adjuvant therapy for the treatment of bladder cancer has not yet been studied.

Overall, the present study demonstrated that high expression of APN plays a key role in the cell proliferation, migration and invasion of bladder cancer cells. Further, we demonstrated that ubenimex could be an excellent inhibitor of APN. The enhancement of autophagy and apoptosis is associated with the inhibition of the Akt signaling pathway. In conclusion, ubenimex could be a desirable adjuvant therapy for the treatment of bladder cancer.

\section{Acknowledgements}

The present study was funded by the National Natural Science Foundation (nos. 81400575 and 81602227), the Science and Technology Development Plan Project of Shandong Province (nos. 2014GSF118144 and 2015GSF118055), the Shandong Provincial Natural Science Foundation (no. ZR2017MH091), the source of our financial support.

\section{References}

1. Antoni S, Ferlay J, Soerjomataram I, Znaor A, Jemal A and Bray F: Bladder cancer incidence and mortality: A global overview and recent trends. Eur Urol 71: 96-108, 2017.

2. Abufaraj M, Shariat SF, Haitel A, Moschini M, Foerster B, Chłosta P, Gust K, Babjuk M, Briganti A, Karakiewicz PI and Albrecht W: Prognostic role of $\mathrm{N}$-cadherin expression in patients with non-muscle-invasive bladder cancer. Urol Oncol 35: 264-271, 2017.

3. Liu CK, Chen Z, Fang J, Xu A, Zhang W and Wang Z: H19-derived miR-675 contributes to bladder cancer cell proliferation by regulating p53 activation. Tumor Biol 37: 263-270, 2016.

4. Liu J, Xu R and Zhao X: Mechanisms for effect of osthole on inhibiting the growth and invasion of bladder cancer cells. Zhong Nan Da Xue Xue Bao Yi Xue Ban 41: 345-352, 2016 (In Chinese).

5. Hashida H, Takabayashi A, Kanai M, Adachi M, Kondo K, Kohno N, Yamaoka Y and Miyake M: Aminopeptidase N is involved in cell motility and angiogenesis: Its clinical significance in human colon cancer. Gastroenterology 122: 376-386, 2002.

6. Mina-Osorio P: The moonlighting enzyme CD13: Old and new functions to target. Trends Mol Med 14: 361-371, 2008.

7. Ishii K, Usui S, Sugimura Y, Yoshida S, Hioki T, Tatematsu M, Yamamoto $\mathrm{H}$ and Hirano $\mathrm{K}$ : Aminopeptidase $\mathrm{N}$ regulated by zinc in human prostate participates in tumor cell invasion. Int $\mathrm{J}$ Cancer 92: 49-54, 2001.

8. Fontijn D, Duyndam MC, van Berkel MP, Yuana Y, Shapiro LH, Pinedo HM, Broxterman HJ and Boven E: CD13/aminopeptidase $\mathrm{N}$ overexpression by basic fibroblast growth factor mediates enhanced invasiveness of 1F6 human melanoma cells. Br J Cancer 94: 1627-1636, 2006.

9. Wang X, Niu Z, Jia Y, Cui M, Han L, Zhang Y, Liu Z, Bi D and Liu S: Ubenimex inhibits cell proliferation, migration and invasion by inhibiting the expression of APN and inducing autophagic cell death in prostate cancer cells. Oncol Rep 35: 2121-2130, 2016.

10. Maiuri MC, Zalckvar E, Kimchi A and Kroemer G: Self-eating and self-killing: Crosstalk between autophagy and apoptosis. Nat Rev Mol Cell Biol 8: 741-752, 2007.

11. Horita H, Frankel AE and Thorburn A: Acute myeloid leukemia-targeted toxin activates both apoptotic and necroptotic death mechanisms. PLoS One 3: e3909, 2008.

12. Yuan $\mathrm{CH}$, Horng CT, Lee CF, Chiang NN, Tsai FJ, Lu CC, Chiang JH, Hsu YM, Yang JS and Chen FA: Epigallocatechin gallate sensitizes cisplatin-resistant oral cancer CAR cell apoptosis and autophagy through stimulating AKT/STAT3 pathway and suppressing multidrug resistance 1 signaling. Environ Toxicol 32: 845-855, 2017.

13. Mujtaba SF, Dwivedi A, Yadav N, Ch R, Kushwaha HN, Mudiam MK, Singh G and Ray RS: Superoxide mediated photomodification and DNA damage induced apoptosis by Benz(a) anthracene via mitochondrial mediated pathway. J Photochem Photobiol B 142: 92-102, 2015.

14. Zhai B, Hu F, Jiang X, Xu J, Zhao D, Liu B, Pan S, Dong X, Tan G, Wei Z, et al: Inhibition of Akt reverses the acquired resistance to sorafenib by switching protective autophagy to autophagic cell death in hepatocellular carcinoma. Mol Cancer Ther 13: 1589-1598, 2014.

15. Wan J, Liu T, Mei L, Li J, Gong K, Yu C and Li W: Synergistic antitumour activity of sorafenib in combination with tetrandrine is mediated by reactive oxygen species (ROS)/Akt signaling. Br J Cancer 109: 342-350, 2013.

16. Tsukamoto H, Shibata K, Kajiyama H, Terauchi M, Nawa A and Kikkawa F: Aminopeptidase N (APN)/CD13 inhibitor, Ubenimex, enhances radiation sensitivity in human cervical cancer. BMC Cancer 8: 74, 2008. 
17. Sekine K, Fujii $\mathrm{H}$ and Abe F: Induction of apoptosis by bestatin (ubenimex) in human leukemic cell lines. Leukemia 13: 729-734, 1999.

18. Liu S, Xie F, Wang H, Liu Z, Liu X, Sun L and Niu Z: Ubenimex inhibits cell proliferation, migration and invasion in renal cell carcinoma: The effect is autophagy-associated. Oncol Rep 33: 1372-1380, 2015

19. Liang W, Gao B, Xu G, Weng D, Xie M and Qian Y: Possible contribution of aminopeptidase N (APN/CD13) to migration and invasion of human osteosarcoma cell lines. Int J Oncol 45: 2475-2485, 2014.

20. Li J, Wang X, Hou J, Huang Y,Zhang Y and Xu W: Enhanced anticancer activity of 5-FU in combination with Bestatin: Evidence in human tumor-derived cell lines and an H22 tumor-bearing mouse. Drug Discov Ther 9: 45-52, 2015.

21. Yamashita M, Wada H, Eguchi H, Ogawa H, Yamada D, Noda T, Asaoka T, Kawamoto K, Gotoh K, Umeshita K, et al: A CD13 inhibitor, ubenimex, synergistically enhances the effects of anticancer drugs in hepatocellular carcinoma. Int J Oncol 49: 89-98, 2016.

22. Nohara S, Kato K, Fujiwara D, Sakuragi N, Yanagihara K, Iwanuma Y and Kajiyama Y: Aminopeptidase N (APN/CD13) as a target molecule for scirrhous gastric cancer. Clin Res Hepatol Gastroenterol 40: 494-503, 2016.

23. Tokuhara $\mathrm{T}$, Hattori $\mathrm{N}$, Ishida $\mathrm{H}$, Hirai $\mathrm{T}$, Higashiyama $\mathrm{M}$, Kodama K and Miyake M: Clinical significance of aminopeptidase $\mathrm{N}$ in non-small cell lung cancer. Clin Cancer Res 12: 3971-3978, 2006.

24. Pang L, Zhang N, Xia Y, Wang D, Wang G and Meng X: Serum APN/CD13 as a novel diagnostic and prognostic biomarker of pancreatic cancer. Oncotarget 7: 77854-77864, 2016.

25. Kehlen A, Lendeckel U, Dralle H, Langner J and Hoang-Vu C: Biological significance of aminopeptidase N/CD13 in thyroid carcinomas. Cancer Res 63: 8500-8506, 2003.
26. Wang SN, Yang SF, Tsai HH, Lee KT and Yeh YT: Increased adiponectin associated with poor survival in hepatocellular carcinoma. J Gastroenterol 49: 1342-1351, 2014.

27. Mizushima N, Levine B, Cuervo AM and Klionsky DJ: Autophagy fights disease through cellular self-digestion. Nature 451: 1069-1075, 2008.

28. Yang ZJ, Chee CE, Huang S and Sinicrope F: Autophagy modulation for cancer therapy. Cancer Biol Ther 11: 169-176, 2011

29. Takeuchi H, Kondo Y, Fujiwara K, Kanzawa T, Aoki H, Mills GB and Kondo S: Synergistic augmentation of rapamycin-induced autophagy in malignant glioma cells by phosphatidylinositol 3-kinase/protein kinase B inhibitors. Cancer Res 65: 3336-3346, 2005.

30. Chen W, Wu J, Shi H, Wang Z, Zhang G, Cao Y, Jiang C and Ding Y: Hepatic stellate cell coculture enables sorafenib resistance in Huh7 cells through HGF/c-Met/Akt and Jak2/Stat3 pathways. Biomed Res Int 2014: 764981, 2014.

31. Song G, Ouyang G and Bao S: The activation of Akt/PKB signaling pathway and cell survival. J Cell Mol Med 9: 59-71, 2005.

32. Yang L, Liu Y, Wang M, Qian Y, Dai X, Zhu Y, Chen J, Guo S and Hisamitsu T: Celastrus orbiculatus extract triggers apoptosis and autophagy via PI3K/Akt/mTOR inhibition in human colorectal cancer cells. Oncol Lett 12: 3771-3778, 2016.

33. Chang CH, Lee CY, Lu CC, Tsai FJ, Hsu YM, Tsao JW, Juan YN, Chiu HY, Yang JS and Wang CC: Resveratrol-induced autophagy and apoptosis in cisplatin-resistant human oral cancer CAR cells: A key role of AMPK and Akt/mTOR signaling. Int J Oncol 50: 873-882, 2017.

This work is licensed under a Creative Commons Attribution-NonCommercial-NoDerivatives 4.0 International (CC BY-NC-ND 4.0) License. 